\title{
TOP QUARK MASS MEASUREMENT AT THE TEVATRON
}

\author{
JOÃO GUIMARÃES DA COSTA \\ FOR THE CDF AND D $\varnothing$ COLLABORATIONS \\ Harvard University, Department of Physics, 17 Oxford St., Cambridge, MA 02138, USA \\ E-mail: guima@huhepl.harvard.edu
}

\begin{abstract}
We report on the latest experimental measurements of the top quark mass by the CDF and D $\varnothing$ Collaborations at the Fermilab Tevatron. We present a new top mass measurement using the $t \bar{t}$ events collected by the DØ Collaboration in Run I between 1994 and 1996. This result is combined with previous measurements to yield a new world top mass average. We also describe several preliminary results using up to $193 \mathrm{pb}^{-1}$ of $t \bar{t}$ events produced in $\bar{p} p$ collisions at $\sqrt{s}=1.96 \mathrm{TeV}$ during the Run II of the Tevatron.
\end{abstract}

\section{Introduction}

The discovery of the top quark in 1995 has once again successfully confirmed the Standard Model (SM) validity. This new quark can now be used to probe the SM limitations. Due to its large mass, the top quark is particularly sensitive to the electroweak symmetry breaking mechanism. The top quark mass, $M_{t}$ is a fundamental parameter in the SM and it is correlated with other SM parameters via electroweak corrections. The precise measurement of $M_{t}$ together with the $W$ boson mass, $M_{W}$ constrains the Higgs boson mass. Currently, the $M_{W}$ is known to less than $0.1 \%$, while the $M_{t}$ uncertainty is about $3 \%$. In Run II, the Tevatron experiments intend to measure its mass with an uncertainty of $3 \mathrm{GeV}$.

In this note, we report on the recent top quark mass measurements by the CDF and $\mathrm{D} \emptyset$ Collaborations using $t \bar{t}$ events collected in $p \bar{p}$ collisions at $\sqrt{s}=1.8 \mathrm{TeV}$ in Run I and at $\sqrt{s}=1.96 \mathrm{TeV}$ in run II (March 2002 - September 2003).

\section{Top Mass Measurement}

At the Tevatron, the top quark is mostly pair produced through quark-antiquark annihilation and gluon-gluon fusion. The SM top quark decays almost exclusively to a $W$ boson and a $b$ quark. Depending on the $W$ boson decays, we categorize the $t \bar{t}$ events as: lepton plus jets, where $t \bar{t} \rightarrow b \bar{b} W W \rightarrow b \bar{b} l \nu q q \prime$ (30\% of $t \bar{t}$ events); or dilepton, where $t \bar{t} \rightarrow$ $b \bar{b} W W \rightarrow b \bar{b} l \nu l \nu$ (5\% of $t \bar{t}$ events). In both cases, $l \equiv$ electron or muon.

The top mass in each event can be reconstructed using the kinematic constraints from the $t \bar{t}$ decay chain. In the lepton plus jets channel, there are 4 jets which corresponds to 12 possible jet-parton combinations and two possible neutrino longitudinal momemtum solutions. This corresponds to 24 solutions for the kinematical fit. To reduce these, one can identify the jets emerging from the $b$-quarks through the measurement of a displaced secondary vertex within the jet, $b$ tagging. One $b$-tag reduces the number of possible solutions to 12 , while two $b$-tags reduces it to 4 solutions. In the dilepton channel, there are two neutrinos which results in an unconstrained system. In this case, extra assumptions need to be made in order to reconstruct the top mass.

The dominant systematic uncertainty, for all top mass measurements, arises from the measurement of the jet energy $(\sim 5-$ $9 \mathrm{GeV})$. Work at both experiments is underway to reduce this uncertainty significantly. The systematic is dominated by the modelling of the calorimeter response and is expected to go down soon due to improvements in the calorimeter simulation and increase of 
the calibration samples size.

\subsection{Improved Run I Measurement}

The DØ Collaboration has recently completed a new top quark mass measurement using $125 \mathrm{pb}^{-1}$ of their lepton plus jets $t \bar{t}$ sample collected between 1994 and 1995 during Run I.

The new method is similar to a method suggested previously ${ }^{1}$. It calculates, as a function of $M_{t}$, the differential probability that the measured variables in each event correspond to $t \bar{t}$ signal. The probability density is written as a convolution of the leadingorder (LO) $t \bar{t}$ production cross-section and any effects from measurement resolution. The detector acceptance and event efficiencies are included through a multiplicative acceptance function that is independent of $M_{t}$. The probability for a background interpretation, $P_{b k g}$ is done in a similar fashion using a matrix element for $W+$ jets production (dominant background source). Finally, the top quark mass is extracted from the maximization of a log-likelihood as a function of $M_{t}$ comprising the set of all candidate events.

The event selection is similar to the original measurement ${ }^{3}$, with a few modifications: (1) There is no requirement of a muon associated with one of the jets, signifying the possible presence of a $b$-quark jet in the final state; (2) Events are required to have exactly four jets to minimize NLO effects when comparing the data with the LO matrix element for $t \bar{t}$ production and decay; (3) A cut on the probability of the events being consistent with background $\left(P_{b k g}<10^{-11}\right)$, to reduce a systematic bias due to large background fraction.

The final result from the maximum likelihood is $M_{t}=180.1 \pm 3.6$ (stat) \pm 3.9 (sys) $\mathrm{GeV} / \mathrm{c}^{2}$. The improvement in statistical significance over the previous $\mathrm{D} \varnothing$ measurement is equivalent to a factor of 2.4 increase in data.
This new measurement was combined with the other Run I measurements, the D $\varnothing$ result in the dilepton sample ${ }^{5}$ and the CDF result in the lepton plus jets sample ${ }^{4}$, to yield a new world average ${ }^{6}$ for the top quark mass, $m_{t}=178.0 \pm 4.3 \mathrm{GeV} / c^{2}$. As a result, the most likely Higgs mass increases from 96 to $114 \mathrm{GeV} / c^{2}$ and the upper limit on the Higgs mass at the $95 \%$ confidence level is raised ${ }^{6}$ from 219 to $260 \mathrm{GeV} / c^{2}$.

\subsection{Lepton Plus Jets Channel in Run II}

Both the CDF and DØ Collaborations have preliminary measurements of the top quark mass using the Tevatron Run II data. In the lepton plus jets decay channel, the CDF Collaboration uses several complementary techniques with a similar event selection. Events are selected by requiring a single central isolated muon or electron with transverse momemtum, $p_{T}>20 \mathrm{GeV} / c$, missing transverse energy greater than $20 \mathrm{GeV}$ indicating the presence of a neutrino, and at least four high$E_{T}$ jets.

The CDF Collaboration has measured the top quark mass with a Dynamical Likelihood Method (DLM) using $165 \mathrm{pb}^{-1}$ of data. This method is similar to the matrix element method used by $\mathrm{D} \emptyset$ for the new Run I top mass result. The likelihood is defined as the differential cross section as a function of the $M_{t}$, multiplied by a transfer function from jets to parton quantities. The method takes into account all possible jet combinations in an event and the likelihood is multiplied event-by-event to derive the top quark mass by the maximum likelihood method.

The measurement is done in the lepton plus jets channels with the standard event selection above. To minimize the contamination from initial and final state radiation the DLM analysis requires exactly four jets in each event, with $E_{T} \geq 15 \mathrm{GeV}$. After selection, there are $22 t \bar{t}$ candidate events in the data with 4.2 events expected from back- 
ground. This method yields a top quark mass of $m_{t}=177.8_{-5.0}^{+4.5}$ (stat) \pm 6.2 (sys) $\mathrm{GeV} / \mathrm{c}^{2}$. This is the most precise top mass measurement so far with Run II data.

The second method used by the CDF Collaboration is a template method ${ }^{4}$ similar to the one used in Run I. In this method, for each possible topological configuration, an event $\chi^{2}$ is computed and minimized. This $\chi^{2}$ takes into account the detector resolution and the $W$ and top quark decay widths. The distribution of the reconstructed mass is compared and fit to Monte Carlo templates with known input $M_{t}$.

This method is applied separately to the data sample with at least one $b$-tag, $m_{t}=$ $174.9_{-7.7}^{+7.1}$ (stat) \pm 6.5 (sys) $\mathrm{GeV} / \mathrm{c}^{2}$ and to the complementary sample without $b$-tags, $m_{t}=179.1_{-9.5}^{+10.5}$ (stat) \pm 8.4 (sys) $\mathrm{GeV} / \mathrm{c}^{2}$. In this case, in order to decrease the backgroud, the jet $E_{T}$ cut for the fourth jet was increased to $22 \mathrm{GeV}$. Once these two measurements are combined the final top mass measurement for the template method is: $m_{t}=176.7_{-5.4}^{+6.0}$ (stat) \pm 7.1 (sys) $\mathrm{GeV} / \mathrm{c}^{2}$.

A third measurement at CDF uses multidimensional templates, Multivariate Template Method. In this case, the jet energy scale is calibrated in the reconstruction of the $W \rightarrow q q \prime$ decay. The method improves the mass resolution by constructing three types of signal templates. One for good permutations, one for bad permutations and a third for incorrect jets. The event kinematic variables are used to determine the probability that the best $\chi^{2}$ results from correct jetparton assignment. The templates are then weighted accordingly. This method increases the signal to background separation by including the sum of the transverse momentum of the four leading jets in the templates. The top mass result is $m_{t}=179.6_{-6.3}^{+6.4}$ (stat) \pm 6.8 (sys) $\mathrm{GeV} / \mathrm{c}^{2}$.

The DØ Collaboration has measured the top quark mass in Run II with an integrated luminosity of $160 \mathrm{pb}^{-1}$. They use two different methods to measure the top quark mass in the lepton plus jets sample. The first method uses templates of signal and background mass spectra. It is similar to the CDF template method described above but without requiring $b$-tagging.

Events are selected by requiring an electron or muon with $p_{T}>20 \mathrm{GeV}$, large missing energy, 3 jets with $p_{T}>20 \mathrm{GeV}$ with at least one extra jet with $p_{T}>15 \mathrm{GeV}$ and finally $E_{T}^{W}=\left|p_{T}^{l}\right|+\left|p_{T}^{\nu}\right|>65 \mathrm{GeV}$. To further discriminate between signal and background, $\mathrm{D} \emptyset$ uses a a discriminat (low bias discriminant $-D$ ) constructed from four topological variables. After the final event selection, there are 87 events in the lepton plus jets sample. The dominant sources of background are $W+$ jets production and multijets. It is predicted that 40 events in the final sample are from background. The top mass obtained by this method is: $m_{t}=$ $170.0 \pm 6.5$ (stat) ${ }_{-6.5}^{+10.2}$ (sys) $\mathrm{GeV} / \mathrm{c}^{2}$.

The second analysis from the $\mathrm{D} \emptyset$ Collaboration uses an approach similar to the one used by DELPHI to measure the $W$ mass $^{8}$ at LEP, the Ideogram method. The mass information, from a kinematic constrained fit is used to construct an event likelihood taking into account all possible jet permutations and neutrino solutions. The low bias discriminant, $D$ is used on an event-by-event basis to estimate the probability that an event is background and no cut on $D$ is applied. The signal term consists of a convolution of the Gaussian resolution functions and a relativistic Breit-Wigner, representing the expected distribution of the average of the two invariant masses of $t$ and $\bar{t}$ in the event. The background is included by a term with the shape of the mass spectra from $W+4$ jets and multijets MC events. Using $191 t \bar{t}$ candidate events with a $68 \%$ background fraction, the measurement gives: $m_{t}=177.5 \pm 5.8$ (stat) \pm 7.2 (sys) $\mathrm{GeV} / \mathrm{c}^{2}$ 


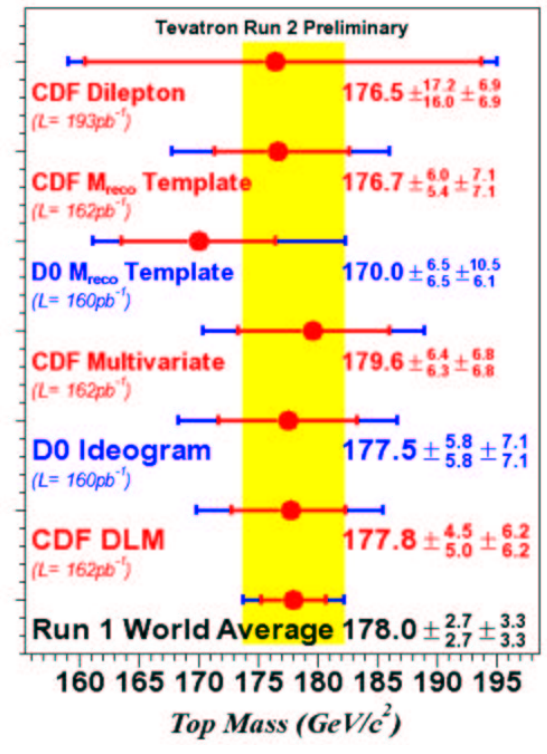

Figure 1. Summary of the top quark mass measurements at the Tevatron.

\subsection{Dilepton Channel in Run II}

The CDF Collaboration has also measured the top quark mass in the $t \bar{t}$ dilepton sample. The technique used in this analysis is also a template method similar to the one described for the lepton plus jets sample. The top mass is reconstructed on an event by event basis, however, the system is under constrained due to the presence of two neutrinos. This problem is solved by introducing an extra condition involving the longitudinal momentum of the $t \bar{t}$ system, $P_{z}^{t \bar{t}}=P_{z}^{t}+P_{z}^{\bar{t}}$.

The analysis uses dilepton $e e, e \mu$ and $\mu \mu$ events. The event selection requires two leptons with $p_{T}>20 \mathrm{GeV} / c$, large missing energy, $\not_{T}>25 \mathrm{GeV}$, and at least two jets with $E_{T}>15 \mathrm{GeV}$. Twelve $t \bar{t}$ candidate pass the reconstruction procedure, to be compared with a background estimation of $2.7 \pm 0.7$. Using these, CDF measures $m_{t}=176.5_{-16.0}^{+17.2}$ (stat) \pm 6.9 (sys) $\mathrm{GeV} / \mathrm{c}^{2}$.

\section{Conclusions}

In this paper, we presented the latest top quark mass measurements by the CDF and $\mathrm{D} \varnothing$ collaborations. The results are summarized in Figure 1.

The latest D $\varnothing$ measurement from Run I data is the most precise measurement from a single experiment. This has resulted in a new more precise top mass world average which has significantly impacted the expectations for the Higgs mass. There are several preliminary measurents from both CDF and $\mathrm{D} \emptyset$ using run II data. The most precise result from run II is the DLM measurement from CDF. These results are expected to be updated soon including data collected until Summer 2004.

The Tevatron is performing very well and large datasets are now becoming available. The top mass measurements will soon be limited by systematic uncertainties. A large effort from both experiment is underway to reduce the systematics, in special the jet energy scale. The goal for run II is to measure the top mass with an uncertainty of $3 \mathrm{GeV}$.

\section{References}

1. Dalitz, R.H. and Goldstein, G.R., Proc. R. Soc. Lond. A 445, 2803 (1999). K. Kondo, J. Phys. Soc. 62, 1177 (1993).

2. Abazov, V.M et al. (DØ Collaboration), Nature 429, 638 (2004).

3. Abbott, B. et al. (DØ Collaboration), Phys. Rev. D 58, 052001 (1998).

4. Affolder, T. et al. (CDF Collaboration), Phys. Rev. D 63, 032003 (2001).

5. Abbott, B. et al. (DØ Collaboration), Phys. Rev. D 60, 052001 (1998).

6. hep-ex/0404010 (2004).

7. lepewwg.web.cern.ch/LEPEWWG/

8. Mulders, B. et al. (DELPHI Collaboration), Int. J. Mod. Phys. A 16S1A, 285 (2001). 